\title{
Differences in unilateral chest press muscle activation and kinematics on a stable versus unstable surface while holding one versus two dumbbells
}

Jeffrey M Patterson, Andrew D Vigotsky, Nicole E Oppenheimer, Erin H Feser

Training the bench press exercise on a traditional flat bench does not induce a level of instability as seen in sport movements and activities of daily living. Twenty participants were recruited to test two forms of instability: using one dumbbell rather than two and lifting on the COR bench compared to a flat bench. Electromyography (EMG) amplitudes of the pectoralis major, middle trapezius, external oblique, and internal oblique were recorded and compared. Differences in range of motion (ROM) were evaluated by measuring an angular representation of the shoulder complex. Four separate conditions of unilateral bench press were tested while lifting on a: flat bench with one dumbbell, flat bench with two dumbbells, COR Bench with one dumbbell, and COR Bench with two dumbbells. The results imply that there are no differences in EMG amplitude or ROM between the COR bench and traditional bench. However, greater ROM was found to be utilized in the single dumbbell condition, both in the COR bench and the flat bench. 


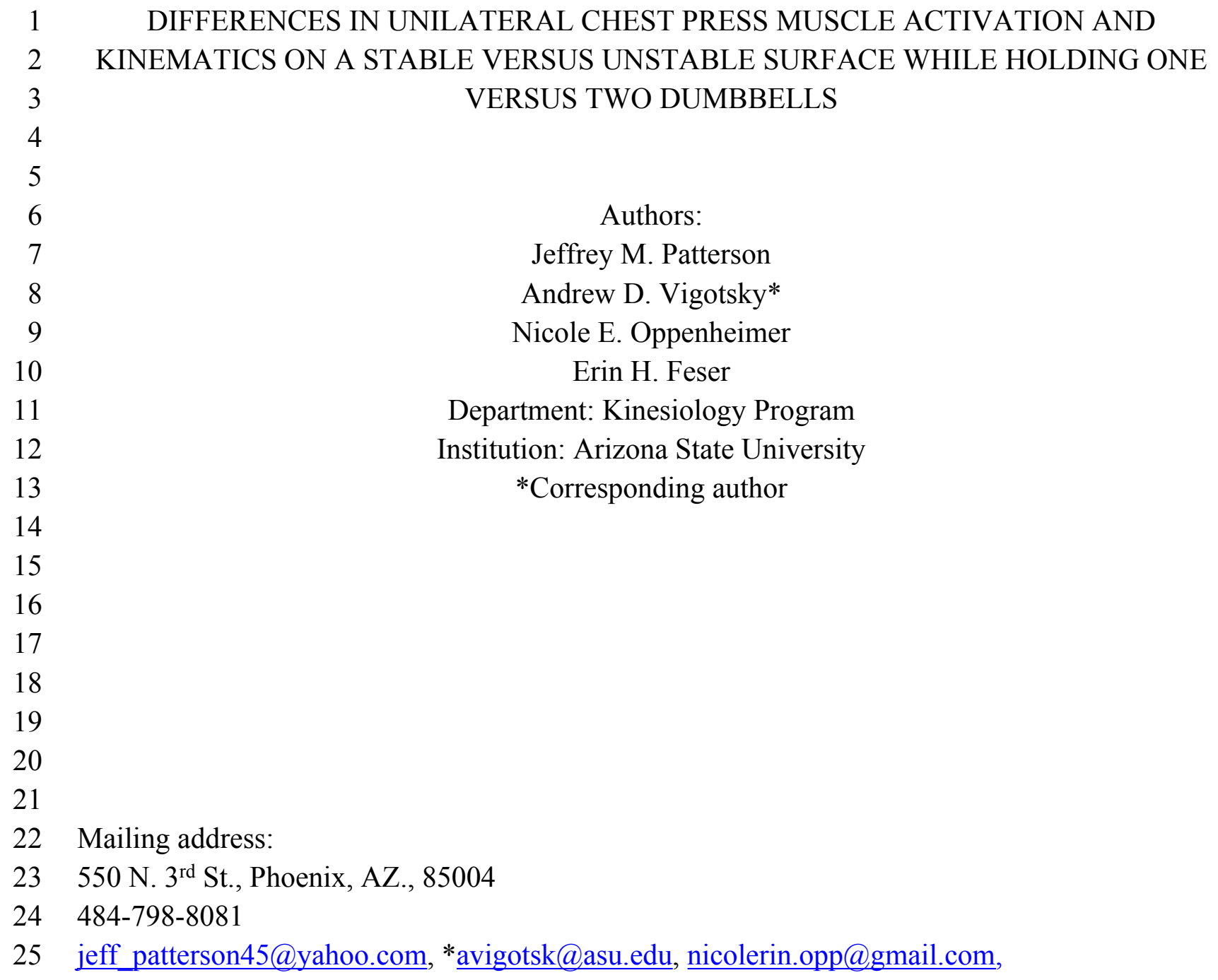

23550 N. $3^{\text {rd }}$ St., Phoenix, AZ., 85004

25 jeff_patterson45@yahoo.com, *avigotsk@asu.edu, nicolerin.opp@,gmail.com,

22 Mailing address:

24 484-798-8081

26 enharper@asu.edu 


\section{ABSTRACT}

29 Training the bench press exercise on a traditional flat bench does not induce a level of instability

30 as seen in sport movements and activities of daily living. Twenty participants were recruited to

31 test two forms of instability: using one dumbbell rather than two and lifting on the COR bench

32 compared to a flat bench. Electromyography (EMG) amplitudes of the pectoralis major, middle

33 trapezius, external oblique, and internal oblique were recorded and compared. Differences in

34 range of motion (ROM) were evaluated by measuring an angular representation of the shoulder

35 complex. Four separate conditions of unilateral bench press were tested while lifting on a: flat

36 bench with one dumbbell, flat bench with two dumbbells, COR Bench with one dumbbell, and

37 COR Bench with two dumbbells. The results imply that there are no differences in EMG

38 amplitude or ROM between the COR bench and traditional bench. However, greater ROM was

39 found to be utilized in the single dumbbell condition, both in the COR bench and the flat bench. 


\section{INTRODUCTION}

41 Both sport and various activities of daily living (ADLs) require individuals to create

42 pushing forces while standing. Most noticeably, this is seen in highly physical sports, like

43 football or wrestling, but the same relative movement is seen when someone pushes a car or

44 moves furniture around their home (Santana et al. 2007). Traditionally, in order to train the

45 muscles involved in these movements, people perform exercises like the bench press. There are,

46 however, inherent problems in using the supine bench press when trying to improve one's ability

47 to create pushing forces when standing.

First, in a supine bench press, either a bar or two free weights being used simultaneously create the resistance in the exercise, making it a bilateral movement. Unfortunately, in most sports and ADLs, movements are predominantly, or entirely, unilateral (Behm et al. 2005). Thus,

52 the principle of specificity is not being fully utilized and the effects of training may not fully crossover.

Secondly, there are many factors that limit one's ability to develop pressing strength while standing, including stability and neuromuscular control of the core musculature, the individual's weight, the base of support the individual utilizes, and the direction in which the pressing motion is performed (Santana et al. 2007). Since a traditional bench press is not performed standing up, these factors are not addressed. In particular, any given individual can usually only produce a standing pushing force of about $40.8 \%$ of their body weight (Santana et al. 2007), whereas it is not uncommon for an individual to be able to lift greater than their body weight in a supine bench press. 
63

64

65

66

67

Additionally, when performing a bilateral, seated pressing motion, the EMG amplitude of core stabilizers, such as the rectus abdominis and external obliques, is reduced significantly when compared to a standing, unilateral pressing motion (Saeterbakken \& Fimland 2012). Since most ADLs and sport actions are performed unilaterally and while standing, training in this method would enhance specificity while also increasing activation and strengthening of the trunk stabilizers (Behm et al. 2005). It can be clearly seen that a supine bench press will not produce the most optimal results for enhanced pressing strength while standing in sport or ADLs.

One final drawback to the bench press is the limitation of a full range of motion (ROM). It is possible that the flat surface of the bench, on which a bench press is completed, may limit an individual's ROM depending on their shoulder width. If individuals are only completing the bench press movement to parallel, they are likely not training through their full ROM. Previous research indicates that training should occur through a person's entire functional ROM (Brown \& Vives 2000; Massey et al. 2005; Pinto et al. 2012).

Because traditional exercises like the bench press do not translate well into standing pressing strength, it is the goal of many researchers today to find ways to make the bench press more similar to an actual movement that would be performed outside of the gym. Researchers have found that exercising on unstable surfaces can increase electromyographic (EMG) amplitude of the core musculature, such as the rectus abdominis (van Dieen et al. 2003), external obliques (Kohler et al. 2010), internal obliques (O'Sullivan 2005), and muscles along the spine; e.g., erector spinae and multifidus (Kohler et al. 2010; O'Sullivan 2005). Furthermore, it has also 
86 been proposed that instead of performing bench press exercises with two dumbbells

87 simultaneously - even if alternating which arm presses as in a unilateral bench press - people

88 should use only one dumbbell at a time (Behm et al. 2010). This is due to that fact that when

89 performing a purely unilateral exercise, the weight creates a torque on the body that the core

90 musculature must counteract, whereas if there is a dumbbell in the offhand it will already

91 counteract that torque with its own torque, thus reducing the work the core must perform.

92

To create an unstable environment, some researchers simply use equipment designed to

94 introduce instability to an exercise. One such piece of equipment is the CÖRTM Bench (COR

95 Bench). The COR Bench is an inflatable 400-denier nylon urethane tube that is supported with a

96 stand much like a normal bench. It is the combination of the rounded surface of the COR Bench

97 and the fact that it is filled with air (i.e., not solid) that create an unstable environment for the

98 individual, which may, in turn, increase both ROM and activation; however, this has yet to be

99 substantiated. Being able to work through a larger ROM and targeting more muscles for

100 stabilization can provide greater neurological and neuromuscular stimulation (Marinković 2011).

101

102 Therefore, the purpose of this study was to examine the following questions: Does

103 performing a chest press on the COR bench elicit greater EMG amplitudes of the core muscles,

104 and possibly the prime movers, when compared to a traditional bench? Will a greater ROM be

105 used on the COR bench than the traditional flat bench? And finally, will a greater ROM be used

106 with one arm when compared with two?

107

108 METHODS 
110 To test whether or not the COR Bench provides a greater stimulus for resistance training,

111 participants performed a one dumbbell and two dumbbell unilateral bench press on both the COR

112 Bench and a traditional bench. Participants were asked to come to the lab to complete two

113 different sessions, the first was to identify the participant's bench press one repetition maximum

114 (1RM) on both the COR Bench and flat bench, and the second was to test muscle activation and

115 the kinematics of their movement during unilateral bench presses with one and two dumbbells on

116 the COR and flat benches.

117

118 Subjects

119 Fifteen males (age, $M=23.1$, range $=20-28$ years; mass, $88.7 \pm 11.9 \mathrm{~kg}$; height, $183.8 \pm$

$1205.6 \mathrm{~cm}$ ) and 7 females (age, $M=20.9$, range = 19-23 years; mass, $56.8 \pm 5.9 \mathrm{~kg}$; height, $167.8 \pm$

$1214.7 \mathrm{~cm}$ ) were recruited to participate over the course of a fall semester; however, the experiment

122 was conducted in a laboratory (controlled environment) with a temperature of $\sim 23^{\circ} \mathrm{C}$.

123 Participants were required to be 18-30 years old, have previous experience in participating in

124 resistance training, be free from current or previous injury that may be aggravated by

125 participating in upper extremity resistance training, and also be familiar with the bench press. All

126 participants were recreationally active. Participants were asked to abstain from training for 24

127 hours prior to testing. Before beginning, all participants completed an Informed Consent and

128 Physical Activity Readiness-Questionnaire. The Institutional Review Board at Arizona State

129 University approved this study and its relevant documents (IRB ID: 1211008510).

130

131 Procedures 
133 by Mayhew et al. (1992). This method was chosen over others because of its accuracy, as

134 determined by a meta-analysis done by LeSuer et al. (1997). Participants' 1RM testing order was

135 randomized. Following, each participant's 1RM could be mathematically predicted; male's 1RM

136 for the flat bench was $65.93 \pm 17.66 \mathrm{~kg}$ and for the COR Bench it was $57.38 \pm 17.40 \mathrm{~kg}$, and

137 female's $1 \mathrm{RM}$ for the flat bench was $18.55 \pm 4.68 \mathrm{~kg}$ and for the COR Bench it was $17.78 \pm 4.47$

$138 \mathrm{~kg}$. All loads are presented as the sum of both dumbbells (total, combined load). Because certain

139 loads could not be created with the dumbbells used in the study, actual weights used were

140 slightly different than the mathematically predicted values. The actual weights used due to

141 dumbbell limitations were as follows: male's 1RM for the flat bench was $65.72 \pm 17.77 \mathrm{~kg}$ and

142 for the COR Bench it was $58.78 \pm 18.76 \mathrm{~kg}$, and female's 1RM for the flat bench was $20.52 \pm$

$1434.88 \mathrm{~kg}$ and for the COR Bench it was $19.44 \pm 4.10 \mathrm{~kg}$.

The second day of testing consisted of the kinematic and EMG analysis. Disposable, selfadhesive, $\mathrm{Ag} / \mathrm{AgCl}$ pre-gelled, bipolar electrodes (Noraxon Product \#272; Noraxon USA Inc.,

147 Scottsdale, AZ), with a diameter of $1 \mathrm{~cm}$ and an inter-electrode distance of $2 \mathrm{~cm}$, were placed on one prime mover muscle (right pectoralis major), one shoulder girdle stabilizer (right middle trapezius), and two muscles associated with core stabilization (left internal and right external obliques) (Konrad 2005). The left internal and right external obliques were selected because they

151 perform ipsilateral and contralateral rotation of the trunk, respectively. Given that the one

152 dumbbell unilateral bench press trials were performed with the dumbbell in the right hand, these

153 muscles should activate to counteract the tendency of the trunk to rotate to the right side. 

removing any hair and abrading and cleaning the skin with alcohol wipes. The EMG signals, collected at $1500 \mathrm{~Hz}$, were analyzed using MyoResearch 3.0 (Noraxon, USA 2012). The collected data was rectified, filtered using a 10-500 Hz bandpass filter, and smoothed using root mean square with a window of $50 \mathrm{~ms}$. Average amplitude was then determined for the duration

160 of each trial.

To assess the kinematics of the participant's movements, reflective markers were placed on the skin over the acromion process of the scapula and the lateral epicondyle of the humerus, allowing for the analysis of ROM during the movement. As the electrodes were reaching a stable impedance condition, the participants engaged in a brief warm-up consisting of 10 arm circles forward, 10 arm circles backward, and 10 wall push-ups. A camera was placed behind the bench

167 to capture the motion of the participant's shoulder complex and their ROM. The shoulder complex ROM (SC ROM) was measured by subtracting the minimum from the maximum 3point angle and averaging the value for the five trials. Each 3- point angle was determined from points at a line held vertical, the acromioclavicular joint, and the lateral epicondyle. This was thought to be the best angular representation of the shoulder complex as a whole (Figure 1). of unilateral bench presses holding two dumbbells on both the COR Bench and the flat bench as well as five repetitions at $70 \%$ of their 1RM of unilateral bench presses holding one dumbbell on 
178 both the COR Bench and flat bench, for a total of 4 separate trials. When they were holding two

179 dumbbells, they completed five repetitions for each arm in an alternating style, starting with both

180 hands raised. In other words, starting with both hands in the air, their right arm came down first,

181 then pushed up, followed by the left arm coming down, then being pushed back up, equaling one

182 repetition per arm. All movements were controlled for speed with a 40 beats per minute tempo

183 metronome ( 2 beats down, 2 beats up), and participants were allowed two minutes rest between 184 sets.

There existed a difference in height between the two benches, so to control for any confounding factors, an elevated footrest was put at the end of the COR Bench to make the heights equal. Additionally, participants were required to keep their feet and legs in similar positions between both 1RM tests and all four experimental trials. This was accomplished by measuring the knee angle (via goniometer) and distance between the participants' feet (via tape measure) while lying on the flat bench, and both of these were hold constant throughout the entire experiment for each participant individually.

\section{Statistical Analysis}

A within-subject experimental design assessing EMG amplitude and movement kinematics was used to test the differences between the COR Bench versus flat bench and one

197 dumbbell versus two dumbbell unilateral bench press. First, normality of the data was checked

198 using the Shapiro-Wilk test. On parametric data, sphericity assumptions were checked using

199 Mauchly's test. Parametric data were analyzed using a repeated-measures analysis of variance

200 (ANOVA). If data were parametric but did not meet sphericity assumptions, Greenhouse-Geisser 
201 corrections to degrees of freedom were applied to the ANOVA. Friedman's test was used to

202 compare within-subject effects across groups for nonparametric data. For parametric data that

203 required pairwise comparisons, two-tailed paired-samples $t$-tests were performed. Non-

204 parametric data that required comparisons were compared using Wilcoxon paired-samples

205 signed-rank tests. A Benjamini-Hochberg procedure was carried out to control for false

206 discovery rate for all pairwise comparisons (Benjamini \& Hochberg 1995; Colquhoun 2014).

207 Alpha was set to $p \leq 0.05$, and $q$-value, or false discovery rate, was also set to 5\%. Parametric

208 effect sizes were calculated by Cohen's $d$ using the formula $d=\frac{M_{d}}{s_{d}}$, where $M_{d}$ is mean

209 difference and $s_{d}$ is the standard deviation of differences (Becker 1988; Morris 2007; Smith \&

210 Beretvas 2009). This method is slightly different than the traditional method of calculating

211 Cohen's $d$, as it calculates the within-subject effect-size rather than group or between-subject

212 effect sizes. Cohen's $d$ was defined as small, medium, and large for 0.20, 0.50, and 0.80,

213 respectively (Cohen 1988). Non-parametric effect-sizes were reported in terms of Pearson's $r($

$\left.214 r=\frac{z}{\sqrt{n}}\right)$. Pearson's $r$ was defined as small, medium, and large for $0.10,0.30$, and 0.50 ,

215 respectively (Cohen 1988). In order to better represent within-subject changes, standard

216 deviations (SD) were calculated based upon normalized data before graphing (Loftus \& Masson

217 1994); although, it should be noted that the within-subject normalization technique does not

218 necessarily represent the pairwise comparisons made, as all four trials were taken into account.

\section{RESULTS}

221 One participant's flat bilateral trial was corrupted (both EMG and ROM) due to a disk

222 writing error, and one participant's middle trapezius EMG signal during the bilateral COR Bench 
223 trial appeared to be invalid, as there were impossibly high amplitudes, potentially due to pressure

224 on the electrode, so these values were not included in the statistical analyses.

225

226

227

228

229

230

231

232

233

234

235

236

237

238

239

240

241

242

243

244

245

Using the calculated $1 \mathrm{RM}$ values, there was a $-11.59 \%$ change in $1 \mathrm{RM}$ value when going from the flat bench to the COR Bench.

The mean EMG $( \pm \mathrm{SD})$ amplitudes for each muscle under each testing condition are displayed in Figure 2. The results of Friedman's test indicate that the differences observed between the four testing conditions in the middle trapezius $\left[\chi^{2}(3)=16.200 ; p=0.001\right]$, external oblique $\left[\chi^{2}(3)=38.556 ; p<0.001\right]$, and internal oblique $\left[\chi^{2}(3)=39.758 ; p<0.001\right]$ were not due to chance alone. However, it cannot be said that the pectoralis major EMG amplitudes between all four testing conditions were statistically different $\left[\chi^{2}(3)=7.444 ; p=0.059\right]$. The results of the pairwise comparisons were used to address the following questions.

\section{[ Insert Figure 2 about here ]}

Is there a difference in EMG amplitude when using one dumbbell on the COR Bench versus one dumbbell on the flat bench?

It cannot be concluded that any differences in EMG amplitude in the muscles analyzed [pectoralis major ( $\mathrm{z}=0.859 ; p=0.5207$; Pearson's $r=0.19)$, middle trapezius $(\mathrm{t}(19)=2.7428 ; p$ $=0.0516$; Cohen's $d=0.61)$, external oblique ( $\mathrm{z}=1.157 ; p=0.4236$; Pearson's $r=0.26)$, or internal oblique $(\mathrm{t}(19)=0.6912 ; p=0.5431$; Cohen's $d=0.15)]$ during the one dumbbell trials on the COR bench versus the two dumbbells on the flat bench were not due to chance alone. 
247 Is there a difference in EMG amplitude when using two dumbbells on the COR Bench versus two 248 dumbbells on the flat bench?

249 It cannot be concluded that any differences in EMG amplitude in the muscles analyzed 250 [pectoralis major $(\mathrm{z}=0.684 ; p=0.5431$; Pearson's $r=0.16)$, middle trapezius $(\mathrm{z}=1.807 ; p=$ 2510.1600 ; Pearson's $r=0.43)$, external oblique $(\mathrm{z}=1.751 ; p=0.1600$; Pearson's $r=0.40)$, or 252 internal oblique $(\mathrm{z}=1.046 ; p=0.4431$; Pearson's $r=0.24)]$ during the two dumbbell trials on 253 the COR Bench versus the two dumbbells on the flat bench were not due to chance alone. 254

Is there a difference in SC ROM between flat vs. COR Bench

256 The mean \pm SD ROM for each testing condition is displayed in Figure 3. The results of 257 the ANOVA indicate that the four testing conditions were statistically different for shoulder 258 complex $\operatorname{ROM}\left[F(3,54)=10.256, p<0.001, \eta^{2}=0.088\right]$. Pairwise comparisons revealed that a 259 statistically greater ROM was utilized in one dumbbell trials when compared to two dumbbells 260 for both the COR bench $(\mathrm{t}(19)=3.826 ; p=0.0066$; Cohen's $d=0.86(0.39,1.32))$ and flat bench $261(\mathrm{t}(18)=4.115 ; p=0.0066$; Cohen's $d=0.94(0.46,1.43))$. However, no statistical differences 262 were observed between the COR bench and flat bench with two dumbbells $(\mathrm{t}(18)=2.509 ; p=$ 2630.0657 ; Cohen's $d=0.58)$ or one dumbbell ( $\mathrm{z}=0.541 ; p=0.5882$; Pearson's $r=0.12)$.

265 [ Insert Figure 3 about here ] 


\section{DISCUSSION}

268

269

270

271

272

273

274

275

276

277

278

279

280

281

282

283

284

285 286

287

288

289

It was hypothesized that the introduction of instability to a unilateral bench press

movement would increase the EMG amplitude of the muscles being analyzed. In other words, an increase in co-contraction should be observed in the conditions with a less stable environment (COR bench) when compared to the conditions with a more stable environment (traditional bench). Additionally, it was hypothesized that an increase in ROM should be observed in the conditions performed on the COR bench due to its curved, narrow, and inflated surface. And lastly, it was hypothesized that participants would utilize a larger ROM with one arm than with two.

When performing the 1RM estimation test (Mayhew et al. 1992) on the flat and COR benches, there existed a $-11.59 \%$ change in $1 \mathrm{RM}$ from the flat bench to COR Bench. This indicates that it would be considerably more difficult to lift a prescribed flat bench workload on the COR Bench. This is important for anyone who plans to use, or train a client/patient on, the COR Bench, as it shows that the instability of the COR Bench is substantial enough to warrant a reduction in the weight being chosen by a factor of about $10 \%$. This is not uncommon, as performing exercises on an unstable surface can reduce the force output of the prime movers (Behm \& Colado 2012; Hubbard 2010; Marinković 2011; Pontillo et al. 2007).

The COR Bench was designed to provide instability to an exercise by not fully supporting the trunk like a traditionally used flat bench. Additionally, its design may allow for a greater ROM. Theoretically, these factors should elicit greater EMG amplitude of the core and 
290 shoulder girdle stabilizers and when performing lifts on the COR Bench when compared to a flat

291 bench. However, in this present study, no statistical differences in EMG amplitude were

292 observed for any muscle (pectoralis major, middle trapezius, external oblique, or internal

293 oblique) when comparing the COR bench to its flat bench counterpart, for both one and two-

294 dumbbell variations. These findings indicate that the COR Bench alone does not add enough

295 instability to truly increase EMG amplitude in either core stabilizer muscles or prime movers

296 analyzed in this study. This is similar to the results of Goodman et al. (2008), who found that

297 there were no differences in EMG amplitude across all muscles being analyzed when performing

298 a chest press on a stable bench and an exercise ball, even when controlling for workload, in

299 addition to Uribe et al. (2010), who found no difference between dumbbell chest press on a

300 bench and exercise ball with $80 \%$ 1RM. Again, however, while it cannot be said that the results

301 were statistically different, there still existed greater EMG amplitude in the COR Bench trials

302 compared to the flat bench trials with medium to large effect sizes. This may be an indicator that

303 future research should be done to further determine the effectiveness of such equipment.

With regards to ROM, it does not appear that the narrower, air compressed surface of the

306 COR bench transfers to greater ROM when compared to that of the traditional bench, for both

307 one- and two-dumbbell variations. However, with both the COR bench and traditional bench,

308 single dumbbell variations utilized a statistically greater ROM than did the two dumbbell

309 variations. Such results suggest that the single dumbbell variation can be utilized to train with

310 greater ROM, but whether these small $\left(\sim 7^{\circ}\right)$, but statistically different, differences in ROM are

311 sufficient to elicit superior training adaptations cannot be said for certain. 
314 present study. Firstly, no specific inclusion or exclusion criteria existed with regards to training

315 age and frequency. It is possible that different responses may have been elicited from those with

316 different training experiences, but this cannot be said for certain. Second, although participants

317 abstained from training for 24 hours prior to the second day of this study, some did complete the

318 testing protocol on the preceding day. Therefore, it is possible that some participants may have

319 been sore or fatigued on the EMG testing day, but because a within-subject design was utilized,

320 participants were familiar with the movement, and the testing protocol was relatively light, it is

321 unlikely that this would have had a substantial impact on the observed outcomes. In addition, the

322 medium to large effect sizes observed for comparisons that did not reach the $a$ priori alpha are

323 indicative that this study may have been underpowered and type-II errors may be present,

324 especially following the correction for multiple comparisons. Lastly, the 1RM estimation

325 protocol utilized in this study has not been validated for the dumbbell bench press, and it is

326 therefore possible that the estimated 1RM differed from participants' actual 1RM.

327 Notwithstanding these limitations, our data are congruent with previous studies (Goodman et al.

328 2008; Uribe et al. 2010).

329

330 CONCLUSION

331 In conclusion, the present study showed that the COR Bench did not prove to be an

332 important of a source of instability, as it failed to elicit both greater EMG amplitude and ROM.

333 Furthermore, it appears that using a single dumbbell may be a better option if larger ROM is

334 desired. More comparative research - including training studies - involving athletes and

335 rehabilitation patients with and without a history of upper extremity injury is warranted. Such 
336 further assessments will promote a better understanding of the other potential implications and

337 advantages of the COR bench.

\section{References}

340

341

342

343

344

345

346

347

348

349

350

351

352

353

354

355

356

357

358

359

360

361

362

363

364

365

366

367

368

369

370

371

372

373

374

375

376

377

Becker BJ. 1988. Synthesizing standardized mean - change measures. British Journal of Mathematical and Statistical Psychology 41:257-278.

Behm D, and Colado JC. 2012. The effectiveness of resistance training using unstable surfaces and devices for rehabilitation. International Journal of Sports Physical Therapy 7:226-241.

Behm DG, Drinkwater EJ, Willardson JM, and Cowley PM. 2010. The use of instability to train the core musculature. Applied Physiology, Nutrition, and Metabolism Physiologie Appliquée, Nutrition et Métabolisme 35:91-108.

Behm DG, Leonard AM, Young WB, Bonsey WA, and MacKinnon SN. 2005. Trunk muscle electromyographic activity with unstable and unilateral exercises. Journal of Strength and Conditioning Research 19:193-201.

Benjamini Y, and Hochberg Y. 1995. Controlling the false discovery rate: a practical and powerful approach to multiple testing. Journal of the Royal Statistical Society Series B (Methodological):289-300.

Brown LE, and Vives D. 2000. Partial or full range of motion during the bench press. Strength \& Conditioning Journal 22:55.

Cohen J. 1988. Statistical power analysis for the behavioral sciences: Routledge Academic.

Colquhoun D. 2014. An investigation of the false discovery rate and the misinterpretation of $p$-values. Royal Society Open Science 1:140216.

Goodman CA, Pearce AJ, Nicholes CJ, Gatt BM, and Fairweather IH. 2008. No difference in 1RM strength and muscle activation during the barbell chest press on a stable and unstable surface. Journal of Strength and Conditioning Research 22:88-94.

Hawley JA. 2008. Specificity of training adaptation: time for a rethink? Journal of Physiology 586:1-2.

Hubbard D. 2010. Is Unstable Surface Training Advisable for Healthy Adults? Strength \& Conditioning Journal 32:64-66.

Kohler JM, Flanagan SP, and Whiting WC. 2010. Muscle activation patterns while lifting stable and unstable loads on stable and unstable surfaces. Journal of Strength and Conditioning Research 24:313-321.

Konrad P. 2005. The ABC of EMG-a practical introduction to kinesiological electromyography. Noraxon Inc, USA.

LeSuer DA, McCormick JH, Mayhew JL, Wasserstein RL, and Arnold MD. 1997. The accuracy of prediction equations for estimating 1-RM performance in the bench press, squat, and deadlift. The Journal of Strength \& Conditioning Research 11:211-213.

Loftus GR, and Masson ME. 1994. Using confidence intervals in within-subject designs. Psychonomic bulletin \& review 1:476-490.

Marinković M. 2011. Muscle strength in unstable conditions. Acta Medica Medianae 50:53-56.

Massey CD, Vincent J, Maneval M, and Johnson JT. 2005. Influence of range of motion in resistance training in women: early phase adaptations. Journal of Strength and Conditioning Research 19:409-411. 
378

379

380

381

382

383

384

385

386

387

388

389

390

391

392

393

394

395

396

397

398

399

400

401

402

403
Mayhew JL, Ball TE, Arnold MD, and Bowen JC. 1992. Relative muscular endurance performance as a predictor of bench press strength in college men and women. The Journal of Strength \& Conditioning Research 6:200-206.

Morris SB. 2007. Estimating effect sizes from the pretest-posttest-control group designs. Organizational Research Methods.

O'Sullivan P. 2005. Diagnosis and classification of chronic low back pain disorders: maladaptive movement and motor control impairments as underlying mechanism. Manual Therapy 10:242255.

Pinto RS, Gomes N, Radaelli R, Botton CE, Brown LE, and Bottaro M. 2012. Effect of range of motion on muscle strength and thickness. Journal of Strength and Conditioning Research 26:2140-2145.

Pontillo M, Orishimo KF, Kremenic IJ, McHugh MP, Mullaney MJ, and Tyler TF. 2007. Shoulder musculature activity and stabilization during upper extremity weight-bearing activities. North American Journal of Sports Physical Therapy 2:90-96.

Saeterbakken AH, and Fimland MS. 2012. Muscle activity of the core during bilateral, unilateral, seated and standing resistance exercise. European Journal of Applied Physiology 112:1671-1678.

Santana JC, Vera-Garcia FJ, and McGill SM. 2007. A kinetic and electromyographic comparison of the standing cable press and bench press. Journal of Strength and Conditioning Research 21:12711277.

Smith LW, and Beretvas SN. 2009. Estimation of the Standardized Mean Difference for Repeated Measures Designs. Journal of Modern Applied Statistical Methods 8:27.

Uribe BP, Coburn JW, Brown LE, Judelson DA, Khamoui AV, and Nguyen D. 2010. Muscle activation when performing the chest press and shoulder press on a stable bench vs. a Swiss ball. Journal of Strength and Conditioning Research 24:1028-1033.

van Dieen JH, Kingma I, and van der Bug P. 2003. Evidence for a role of antagonistic cocontraction in controlling trunk stiffness during lifting. Journal of Biomechanics 36:1829-1836. 
1

Angular Representation of the Measurement of Shoulder Complex Range of Motion

Differences in ROM evaluated by measuring angular representation of the shoulder complex: a 3-point angle determined from points at a line held vertical, the acromioclavicular joint, and the lateral epicondyle.

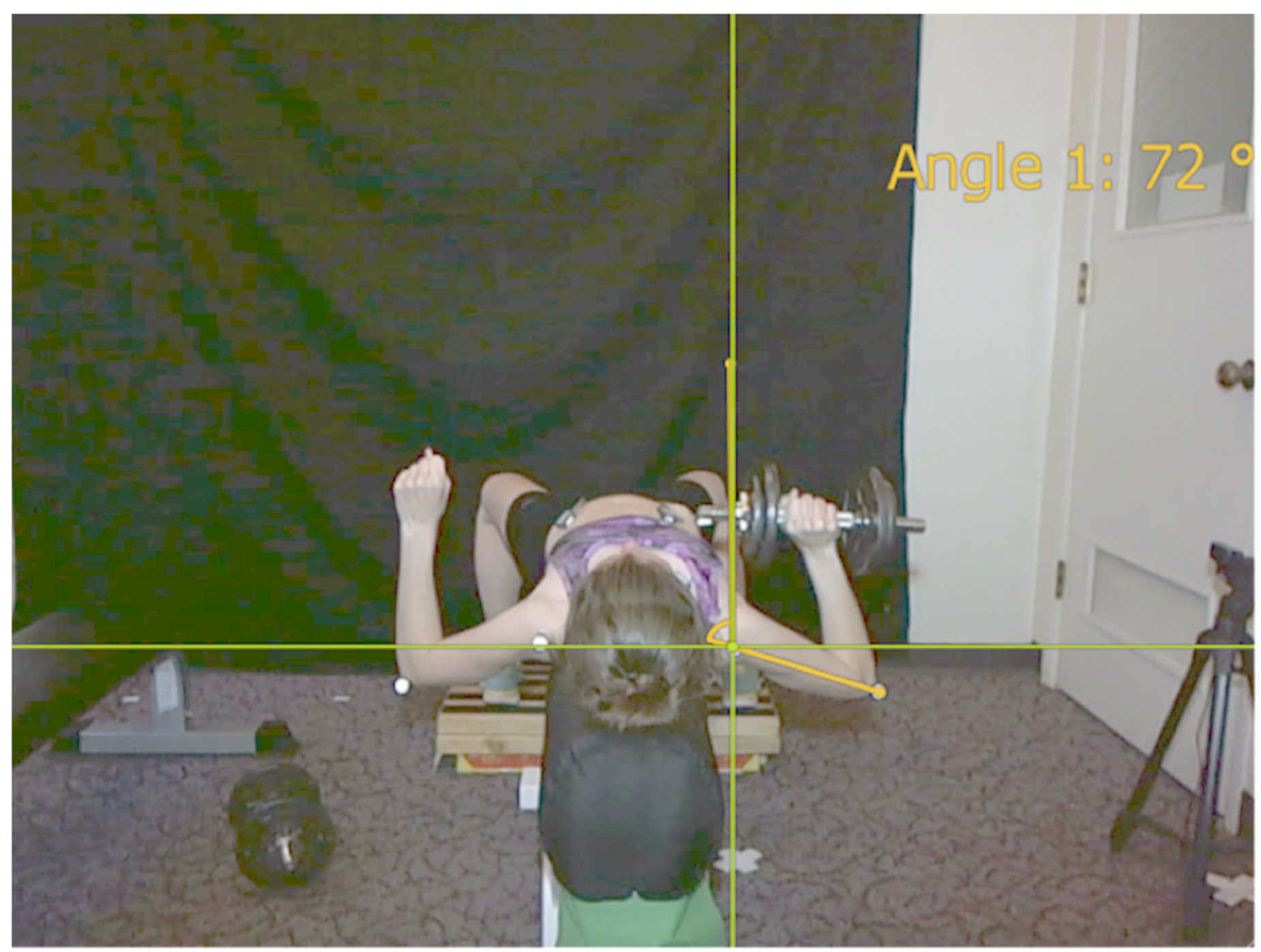


Figure 2 (on next page)

Mean EMG amplitude ( \pm SD) in all 4 trials across all participants. 


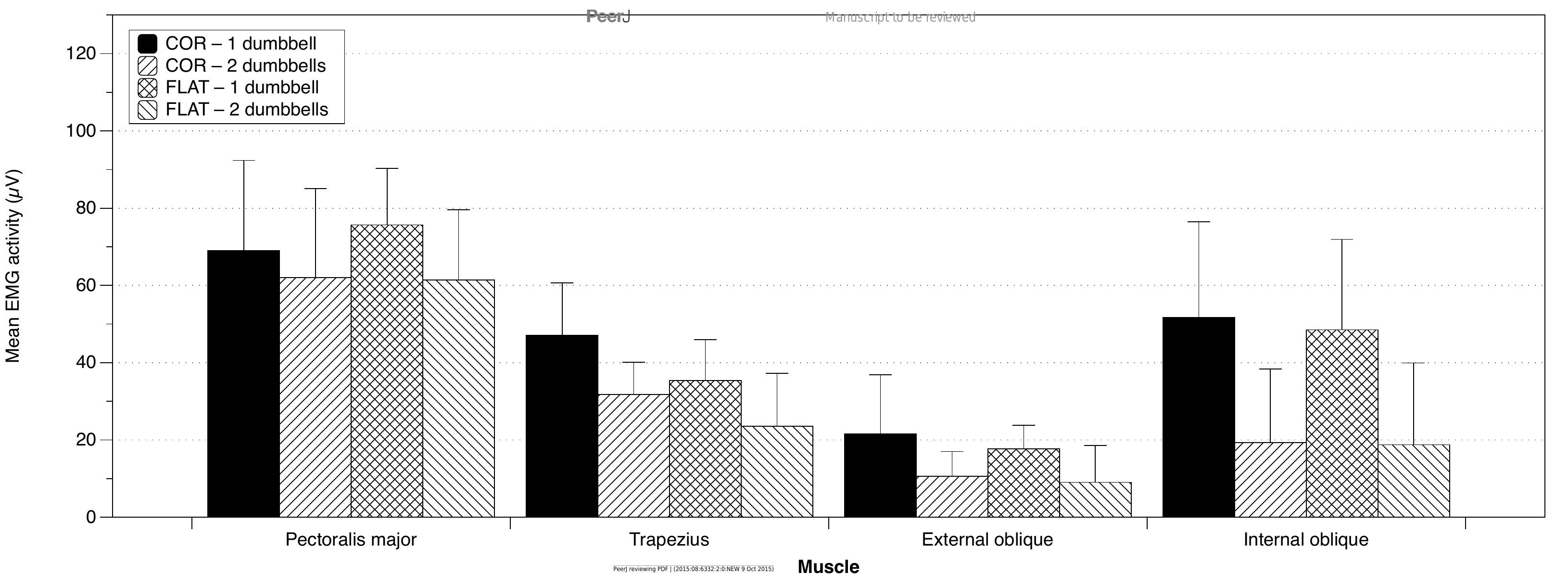




\section{Figure 3 (on next page)}

Mean ( \pm SD) shoulder complex ROM in all 4 trials across all participants.

${ }^{*}$ ) denotes statistically greater shoulder complex ROM than using two dumbbells on the COR bench. $(\dagger)$ denotes statistically greater shoulder complex ROM than using two dumbbells on the flat bench 


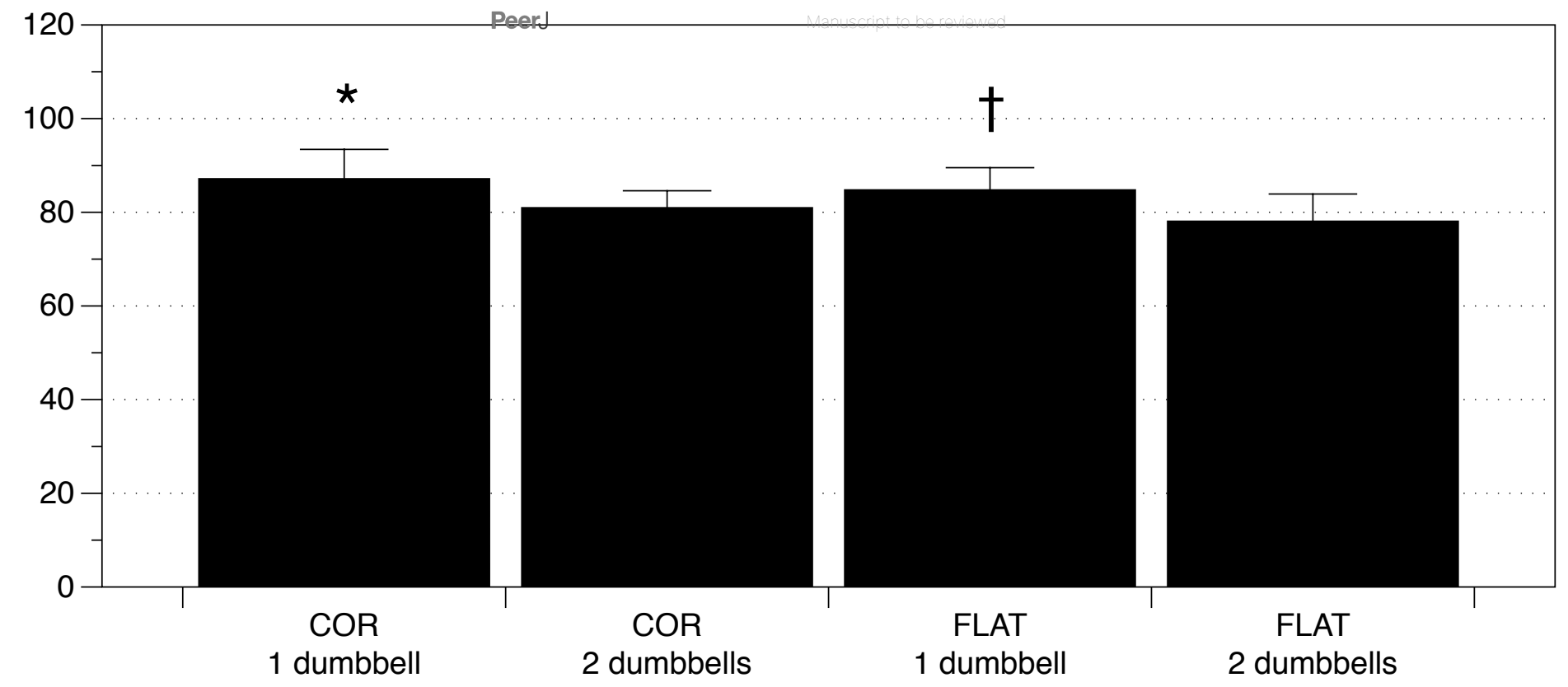

